Chirurg 2015 $\cdot 86: 287$

DOI 10.1007/s00104-015-3001-5

Online publiziert: 28. Februar 2015

๑) Springer-Verlag Berlin Heidelberg 2015

T. Franzke $\cdot$ J. Jähne

Klinik für Allgemein- und Viszeralchirurgie, Schwerpunkt für endokrine und onkologische Chirurgie,

Sektion für Adipositaschirurgie, Diakoniekrankenhaus Henriettenstiftung Hannover

\title{
Bedeutung präoperativer Risikofaktoren für die Morbidität nach Ösophagusresektionen
}

\section{Ergebnisse}

\section{Diskussion}

\section{Methodik und Patienten}

Zwischen 2001 und 2011 wurden $128 \mathrm{~Pa}$ tienten am Universitätsklinikum Lausanne aufgrund eines Ösophaguskarzinoms operiert. Die Daten wurden prospektiv erfasst und ausgewertet. Als potenziell beeinflussbare Risikofaktoren wurden der Ernährungszustand (Body-MassIndex [BMI], Serumalbumin) sowie ein Alkohol- und Nikotinabusus untersucht.
Die Einschlusskriterien wurden von 93 Patienten erfüllt. Bei 71 Patienten wurde ein Magenhochzug mit thorakalem Anschluss und bei 16 Patienten mit zervikalem Anschluss durchgeführt. 6 Patienten erhielten eine transhiatale Gastrektomie. Die Gesamtkomplikationsrate lag bei $81 \%$ (56\% minor; $18 \%$ major). Die Mortalitätsrate betrug $6 \%(n=6)$. Am häufigsten traten pulmonale Probleme $(55 \%, n=59)$, gefolgt von kardiovaskulären Ereignissen (35\%; $n=38)$ und Anastomoseninsuffizienzen (18\%; $n=19)$ auf. In der multivariaten Analyse konnten weder ein erniedrigter BMI und die Hypalbuminämie noch ein erhöhter Alkoholkonsum als unabhängige Risikofaktoren für postoperative Komplikationen bestätigt werden. Der Nikotinabusus zeigte sich demgegenüber als signifikanter Risikofaktor für Majorkomplikationen $(\mathrm{p}=0,002)$. Bei der Analyse des simultanen Auftretens von zwei respektive mehreren der untersuchten Risikofaktoren zeigte sich eine signifikante Beziehung zur Gesamtkomplikationsrate. 83\% der Patienten mit Majorkomplikationen hatten wenigstens zwei der untersuchten Risikofaktoren.

lungsparameter des Ernährungszustandes zu bemängeln.

Die von den Autoren untersuchten Risikofaktoren spiegeln die typische Konstellation von Patienten mit einem Ösophaguskarzinom wider und sind zugleich auch entscheidend an der Karzinogenese beteiligt. Daher ist es nicht verwunderlich, dass diese Faktoren bzw. deren simultanes Auftreten auch einen negativen Einfluss auf das direkte postoperative Outcome haben.

Ob durch eine präoperative Umstellung der Lebensgewohnheiten (Nikotinund Alkoholkarenz) und eine Verbesserung der Ernährungssituation tatsächlich ein positiver bzw. protektiver Effekt erzielt werden kann, muss in weiteren Studien geklärt werden. Für den klinischen Alltag stellt dies die größte Schwierigkeit dar, da diese spezielle Vorbereitung nicht nur eine interdisziplinäre, sondern auch eine intersektorielle Herausforderung bedeutet, einer adäquaten Patientencompliance bedarf und darüber hinaus einen längeren Zeitraum in Anspruch nimmt. In diesem Kontext ist gegenwärtig unklar, wie lange eine Alkohol- und Nikotinkarenz nötig ist, um eine wirkliche Verbesserung der Risikosituation zu realisieren.

\section{Korrespondenzadresse}

Die vorliegende Studie verdeutlicht, dass Ösophagusresektionen als Hochrisikooperationen zu bewerten sind. Die Komplikationsrate von $81 \%$ erscheint realistisch, allerdings ist die geringe Fallzahl zu berücksichtigen (etwa 12 Resektionen/ Jahr). Des Weiteren sind das retrospektive Design und die aus unserer Sicht ungenügende Anwendung des BMI in Kombination mit dem Serumalbumin als Beurtei-

\section{Dr. T. Franzke}

Klinik für Allgemein- und Viszeralchirurgie, Schwerpunkt für endokrine und onkologische Chirurgie, Sektion für Adipositaschirurgie, Diakoniekrankenhaus Henriettenstiftung Hannover,

Marienstr. 72-90, 30171 Hannover

Interessenkonflikt. T. Franzke und J. Jähne geben an dass kein Interessenkonflikt besteht.
Timm.Franzke@ddh-gruppe.de 